\title{
A REGULAR FORM OF THE SCHMID LAW. APPLICATION TO THE AMBIGUITY PROBLEM
}

\author{
Mayeul ARMINJON \\ Institut de Mécanique de Grenoble, B.P. 53 X, \\ 38041 Grenoble cédex, France
}

\section{INTRODUCTION}

The classical Schmid law postulates that a critical value $\tau_{\mathbf{k}}^{\mathrm{c}}$ of the resolved shear stress $\tau_{k}$ must be reached, if a given slip system $k$ is currently activated in a deformed crystal - i.e., if the corresponding shear rate $\dot{\gamma}_{k}$ is not zero. The experimental verifications are approximate : the critical values cannot be defined with the same accuracy as the measured shear stresses and strains; this is scarcely questionable as regards the (not allowed) variation of the measured $\tau_{k}^{c}$ with the crystal orientation in single slip situations ${ }^{1}$. Hence, even though the Schmid law has proved to be an extremely useful tool, it should not be considered as intangible. Since it may lead to undeterminacies, e.g. to ambiguous stress states and lattice spins when the plastic strain rate is known ${ }^{2-4}$, modifications of the Schmid law have in fact already been proposed, following the way introduced by Hutchinson 5 . In 5 , the strain rate sensitivity of the creep behaviour was taken into account by assuming a power-law relationship between $\tau_{k}$ and $\dot{\gamma}_{k}$; the obtained constitutive relation for a crystal was analysed and applied to calculate global stresses in isotropic polycrystals. This visco-plastic regularization of the Schmid law has also been used for textured polycrystals, in the original form ${ }^{6-7}$ or in the form of a "bilinear relation" between $\tau_{k}$ and $\dot{\gamma}_{k} 8$. While its application to hot deformation is natural 5,7 , it is less obvious and remains discussed ${ }^{9}$ for the case of cold deformation at ordinary rates, where the experimental rate-sensitivity is very low.

Here, an analysis of the classical Schmid law is presented and a regular (power-law) form ${ }^{10}$ is proposed, solving ambiguities within the frame of rate-independant plasticity. A comparison is also made between the limit behaviours of the proposed and viscoplastic (power-law) regularizations, as the regularization parameter $1 / \mathrm{n}$ tends towards zero.

\section{ANALYSIS OF THE SCHMID LAW}

\subsection{The classical Schmid law}

The plastic strain-rate tensor $\mathbf{D}$ in the considered, homogeneously deformed crystal, is assumed to be a linear combination of simple shears occurring on crystallographic planes, with normal $\mathbf{n}_{\mathbf{k}}$, in crystallographic directions $\mathbf{g}_{\mathrm{k}}$ (with $\left.\mathbf{g}_{\mathrm{k}} \cdot \mathbf{n}_{\mathrm{k}}=0\right)$ : 


$$
\mathbf{D}=\sum_{\mathbf{k}=1}^{\mathrm{K}} \dot{\gamma}_{\mathbf{k}} \cdot \mathbf{s}\left(\mathbf{g}_{\mathrm{k}} \times \mathbf{n}_{\mathrm{k}}\right)=\mathbf{D}(\dot{\boldsymbol{\gamma}}), \dot{\boldsymbol{\gamma}}=\left(\dot{\gamma}_{\mathbf{k}}\right)_{1 \leq \mathrm{k} \leq \mathrm{K}}
$$

where $\mathrm{K}$ is the total number of slip systems in the crystal $(\mathrm{K}=12$ for f.c.c. crystals), $\mathbf{g}_{\mathbf{k}} \times \mathbf{n}_{\mathbf{k}}=\mathbf{G}_{\mathbf{k}}$ denotes the shear tensor with unit shear rate, on system (k) : $\mathbf{G}_{\mathbf{k}} \cdot \mathbf{x}=\left(\mathbf{x} \cdot \mathbf{n}_{\mathbf{k}}\right) \mathbf{g}_{\mathbf{k}}$, and $\mathbf{s}_{\mathbf{G}}$ is the symmetric part of a tensor $\mathbf{G}$. The Schmid law lays down two rules governing the selection of the active slip systems $\mathbf{k}$ and the corresponding values $\dot{\gamma}_{k} \neq 0$

(i) for any system $\mathrm{k}$ (whether active or not), the resolved shear stress

$\tau_{\mathbf{k}}(\boldsymbol{\sigma})=\left(\boldsymbol{\sigma} \cdot \mathbf{n}_{\mathbf{k}}\right) \cdot \mathbf{g}_{\mathbf{k}}=\boldsymbol{\sigma}:\left(\mathbf{g}_{\mathbf{k}} \times \mathbf{n}_{\mathbf{k}}\right)=\boldsymbol{\sigma}: \mathbf{G}_{\mathbf{k}}$

cannot exceed a "critical shear stress" $\tau_{k}^{c}:\left|\tau_{k}\right| \leq \tau_{k}^{c}$ (here $\sigma$ is the stress tensor); moreover $\left|\tau_{\mathbf{l}}\right|=\tau_{\mathbf{k}}^{c}$ for active slip systems.

(ii) the shear stress and shear rate have the same sign : $\tau_{\mathrm{k}} \cdot \dot{\gamma}_{\mathrm{k}} \geq 0$. Thus $\tau_{\mathrm{k}} \cdot \dot{\gamma}_{\mathrm{k}}=\tau_{\mathrm{k}}^{\mathrm{c}} \cdot \dot{\gamma}_{\mathrm{k}} \mid>0$ for active slip systems.

The first requirement defines the yield criterion :

$$
f(\sigma)=f_{1}\left(\tau_{1}(\sigma)\right)=1
$$

where

$$
f_{1}(\tau)=f_{1}^{(\infty)}(\tau)=\operatorname{Max}\left\{\mid \tau_{k} / / \tau_{k}^{c} ; 1 \leq k \leq K\right\}
$$

and $\tau=\tau_{1}(\sigma)=\left(\tau_{k}(\sigma)\right)_{1 \leq k \leq K}$ is the shear stress vector associated with $\sigma$

2.2. The yield surface in $\sigma$ - or $c$ - space

The "large" surface $\Sigma_{1}^{\prime}$ with equation $f_{1}(\tau)=1$ (eqn. (4)) may be defined in the $\mathrm{K}$ - dimensional $\tau$ - space : it is simply a rectangular parallelepiped with sides $2 \tau_{k}^{c}(1 \leq k \leq K)$, centered at $\tau=0$. However, only the points $\tau=\tau_{1}(\sigma)$ which may be associated with a stress tensor $\sigma$ have a physical meaning. Since $\mathbf{n}_{\mathbf{k}} \cdot \mathbf{g}_{\mathbf{k}}=\mathbf{0}$, the resolved shear stresses $\tau_{k}(\sigma)$ and thus the vector $\tau_{1}(\sigma)$ do not depend on the pressure $p=-\left(\sigma_{11}+\sigma_{22}+\sigma_{33}\right) / 3$; hence only deviatoric stress tensors (i.e. such that $p=0$ ) have to be considered. Moreover, the linear mapping $\tau_{1}$ from the 5 -dimensional space $S_{0}$ of deviatoric stresses into (but not onto) the $\tau$-space, is one-to-one for the case of common cubic crystals. The physically relevant part $\Sigma_{1}$ of the large surface $\Sigma_{1}^{\prime}$ is thus the section of $\Sigma_{1}^{\prime}$ by the 5-dimensional linear subspace $\tau_{1}\left(S_{0}\right)$ of the $\tau$-space. The ordinary yield surface $\Sigma$ is deduced from $\Sigma_{1}$ by the inverse linear mapping $\tau_{1}{ }^{-1}$ (only defined on 


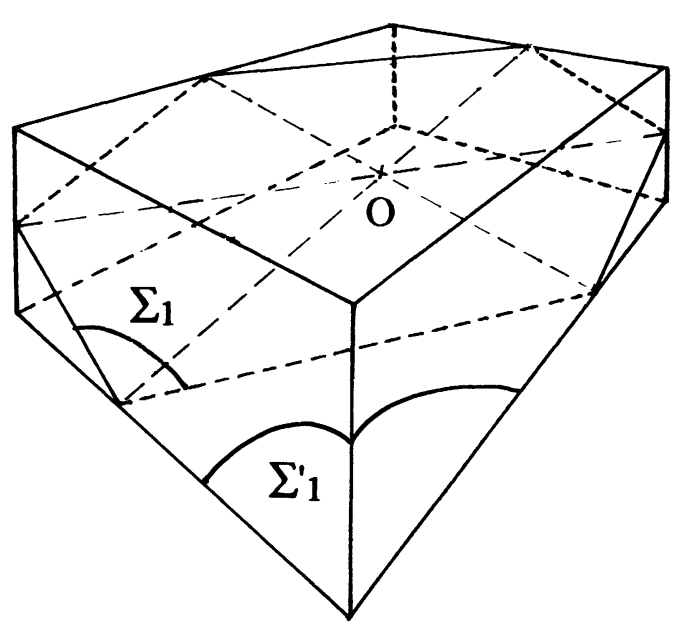

$\tau_{1}\left(S_{0}\right)$ ). Thus the geometrical appearance of $\sum$ is essentially that of $\Sigma_{1}$ (up to a kind of "homogeneous strain"). In particular, the number of corners, ridges, faces ....as well as the order of the corners, are the same in $\Sigma$ and $\Sigma_{1}$. On the other hand, these numbers and orders are not the same in $\Sigma_{1}^{\prime}$ and in its section $\Sigma_{1}$ by $\tau_{1}\left(S_{0}\right)$, though the very

Fig. 1 - Perspective view of $\Sigma_{1}^{\prime}$ and $\Sigma_{1}$ for a 3-D $\tau$-space and a 2-D $\sigma$-space

existence of ridges and corners on $\sum$ is clearly inherited from the large parallelepiped $\Sigma_{1}^{\prime}$ (fig. 1).

\subsection{Rule of the signs and normality rule}

The "rule of the signs" : $\tau_{\mathrm{k}} \cdot \dot{\gamma}_{\mathrm{k}}=\tau_{\mathrm{k}}^{\mathrm{c}} \cdot \dot{\gamma}_{\mathrm{k}} \mid$ for all $\mathrm{k}$, (trivial when $\dot{\gamma}_{\mathrm{k}}$ $=0$ ), means exactly that the shear rate vector $\dot{\gamma}=\left(\dot{\gamma}_{k}\right)_{1 \leq k<K}$ lies within the cone of outer normals to the large surface $\Sigma_{1}^{\prime}$ at $\tau=\left(\tau_{\mathrm{k}}\right)$. Indeed, the latter condition is equivalent to say that :

$$
\tau^{*} \cdot \dot{\gamma} \leq \tau \cdot \dot{\gamma} \text { if } \tau^{*} \text { is on } \Sigma_{1}^{\prime} \text { or within it }
$$

Thus $\tau$ renders $\tau^{*} . \dot{\gamma}$ a maximum among the vectors $\tau^{*}$ satisfying $\left|\tau_{\mathbf{l}}^{*}\right| \leq \tau_{k}^{c}$ for all $k$. The value of this maximum is characterized by :

$$
\sum_{\mathbf{k}=1}^{\mathrm{K}} \tau_{\mathbf{k}} \cdot \dot{\gamma}_{\mathbf{k}}=\sum_{\mathbf{k}=1}^{\mathrm{K}} \tau_{\mathbf{k}}^{\mathbf{c}}\left|\dot{\gamma}_{\mathbf{k}}\right|=\dot{\mathrm{W}}_{2}(\dot{\boldsymbol{\gamma}})
$$

which proves the stated equivalence. Since $\Sigma_{1}$ is a part of $\Sigma_{1}^{\prime}, \dot{\gamma}$ is also a normal to $\Sigma_{1}$ at $\tau$. Now, if $\tau^{*}=\tau_{1}\left(\sigma^{*}\right)$, we have from (1) and (2):

$$
c^{*} \cdot \dot{\gamma}=\sigma^{*}: D(\dot{\gamma})
$$

and the normality of $\dot{\gamma}$ to $\Sigma_{1}$ is thus equivalent to the classical maximum work principle ${ }^{11}$ i.e. to the normality of $D$ to $\Sigma$ in $\sigma$-space.

Hence, the rule of the signs is actually stronger than the classical normality, since it is much more restrictive to assume that $\dot{\gamma}$ is normal to 
$\Sigma_{1}^{\prime}$ than to $\Sigma_{1}$ (unless the normal to $\Sigma_{1}$ were assigned to be within $\tau_{1}\left(S_{0}\right)$ : but this is not the case for the Schmid law).

\section{THE PROPOSED REGULAR FORM AND ITS PROPERTIES}

\subsection{The proposed regular form, solving ambiguity problems}

The yield criterion in $\tau$-space (4) associated with the classical Schmid law is replaced by the following one :

$$
f_{1}^{(n)}(\tau)=\sum_{k=1}^{K}\left(\tau_{l} / / \tau_{k}^{c}\right)^{n}
$$

in the right-hand side of which $\mathrm{n}$ is an exponent. For any $\mathrm{n}>1$, the obtained large yield surface $\Sigma_{1}^{\prime(n)}$ is regular (i.e. has a unique normal $\dot{\gamma}$ at any point $\tau$ ) and strictly convex (i.e. does not contain any segment) : so also are its section $\Sigma_{1}^{(n)}$ by the linear domain $\tau_{1}\left(S_{0}\right)$ of the attainable vectors $\tau$, and the yield surface in $\sigma$-space, $\Sigma^{(\mathrm{n})}=\tau_{1}^{-1}\left(\Sigma_{1}^{(\mathrm{n})}\right)$. Moreover , the true vector $\dot{\gamma}$ is this time postulated to be normal to $\Sigma_{1}^{\prime(n)}$ (the large one), instead of prescribing the sign of the shears. Thus if $\sigma$ is given, $\dot{\gamma}$ is the unique normal to $\Sigma_{1}^{\prime(n)}$ (eqn. 8) at $c=\tau_{1}(\sigma)$ :

$$
\dot{\gamma}_{k}=\dot{\lambda} \frac{\partial f_{1}^{(n)}}{\partial \tau_{k}}=n \dot{\lambda} \frac{\operatorname{sgn}\left(\tau_{k}\right)}{\tau_{k}^{c}}\left(\mid \tau_{k} / / \tau_{k}^{c}\right)^{n-1}
$$

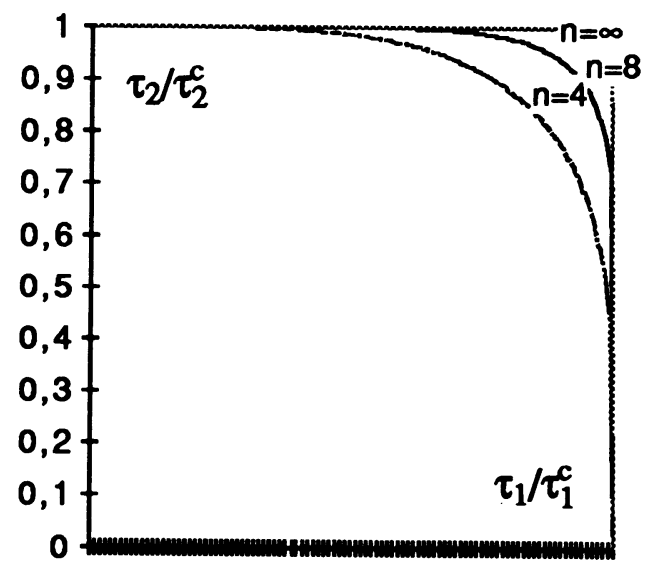

Fig. 2 - The classical $(n=\infty)$ and regular yield surface in a 2-D $\tau$-space, in

Fig. 2 - The classical ( $\mathrm{n}$
reduced shear stresses. $( \pm 1)$ and $\lambda$ is the "plastic multiplier" of classical plasticity $: \dot{\lambda}$ is arbitrary if $\sigma$ alone is given. Conversely, if $D$ is given, $\sigma$ is given by the maximum work principle, i.e. $\sigma$ is the unique point of $\Sigma$ such that $D$ is normal to $\Sigma$ at $\sigma$ (the uniqueness comes from the strict convexity of $\Sigma$ ). The shear rates are then determined by (9) and (1). where $\operatorname{sgn}\left(\tau_{k}\right)$ is the sign of $\tau_{k}$ 
Here again the normality in $\sigma$-space follows immediately from (7) and the normality in $\tau$-space. For sufficiently large $n$, the regular yield criterion (8) is arbitrarily near of the classical one (4), moreover the yield surface $\Sigma_{1}^{\prime(n)}$ is interior to $\Sigma_{1}^{\prime}$ and tangent to it at the points $\tau(1)$, with components $\tau_{k}^{(1)}=\delta_{k 1} \tau_{1}^{c}$ in the $\tau$-space (fig. 2). Hence $\Sigma(n)$ is arbitrarily near of $\Sigma$ and interior to it, but it is not tangent to it since for cubic crystals no stress can make all the $\tau_{k}$ 's nil but one, i.e. the points $\tau^{(l)}$ are not attainable.

\subsection{Solution procedure for polycrystal models}

In the Taylor model as well as in the relaxed Taylor theory $2-4,12$ or in the more general model ${ }^{13-14}$, the primary (input) variable is $\mathrm{D}$, the (plastic) strain-rate of the considered crystal (in the Taylor model this is also the macroscopic strain-rate ; in the relaxed Taylor theory, only a subset $\left(D_{i j}(i, j) \in I J\right)$ of the $D$ components is needed, and assumed equal to the macro-components 2,4 ; in 13-14 $\mathrm{D}$ lies within a prescribed neighborhood of the macro-tensor and the distribution of the D's for the different crystals minimizes the macroscopic plastic work). First, $\sigma$ is (uniquely) determined by using the maximum work principle :

$$
\begin{gathered}
\dot{W}(D)=\sigma: D=\operatorname{Max}\left\{\sigma^{*}: D ; f^{(n)}\left(\sigma^{*}\right) \leq 1\right\}=\operatorname{Max} \phi_{D}\left(\sigma^{*}\right) \\
\phi_{D}\left(\sigma^{*}\right)= \begin{cases}\sigma^{*}: D \text { if } f^{(n)}\left(\sigma^{*}\right) \equiv f_{1}^{(n)}\left(\tau_{1}\left(\sigma^{*}\right)\right) \leq 1 \\
0 & \text { otherwise }\end{cases}
\end{gathered}
$$

Then, the direction of the shear rate vector $\dot{\gamma}$ follows analytically (eqn. (9) with $\dot{\lambda}=1$ ). The multiplier $\dot{\lambda}$ is finally found from (1). In the relaxed Taylor theory, $\sigma^{*}: D$ has only to be replaced by $\sum_{(j, j) \in U} \sigma_{i j}^{*} D_{i j} 2,4$. It is easy to show that the minimum work principle ${ }^{11}$ also holds ${ }^{10}$, namely :

$$
\begin{aligned}
& \dot{\mathrm{W}}(\mathbf{D})=\dot{\mathrm{W}}_{2}(\dot{\gamma})=\operatorname{Min}\left\{\dot{\mathrm{W}}_{2}\left(\dot{\gamma}^{*}\right) ; \mathbf{D}\left(\dot{\gamma}^{*}\right)=\mathbf{D}\right\} \\
& \dot{\mathrm{W}}_{2}\left(\dot{\gamma}^{*}\right)=\tau\left(\dot{\gamma}^{*}\right) \cdot \dot{\gamma}^{*}=\operatorname{Max}\left\{\tau^{* *} \cdot \dot{\gamma}^{*} ; \mathrm{f}_{1}^{(\mathrm{n})}\left(\tau^{* *}\right) \leq 1\right\}
\end{aligned}
$$

However (12) does not seem to provide an easy procedure because $\dot{W}_{2}\left(\dot{\gamma}^{*}\right)$ does not depend analytically on $\dot{\gamma}^{*}$, contrary to the classical case (eqn. (6)).

Consider now the case where the stress-rate $\hat{\sigma}$ (corotational to the lattice ${ }^{15}$ ) is the primary variable ${ }^{16}$. The current stress $\sigma$ is then known 
from previous incremental calculations, and determines the direction of $\dot{\gamma}$ and D (eqns. (9) - (1)). Since the yield condition $\mathrm{f}_{1}^{(\mathrm{n})}(\tau)=1$ (eqn. (8)) must hold during the whole considered time increment, its time derivative (including the variation of the $\tau_{k}^{c}$ 's as well as that of the $\tau_{k}$ 's) is nil ${ }^{15}$. In the case of our regular form and when each $\dot{\tau}_{\mathbf{k}}^{c}$ depends linearly on the $\dot{\gamma}_{1}{ }^{15}$, this gives

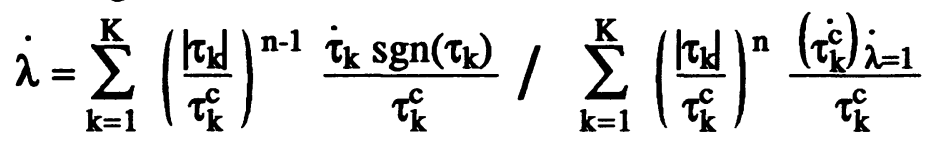

under the loading condition $\dot{\tau} . \partial \mathrm{f}_{1}^{(\mathrm{n})} / \partial \tau=$ [numerator of (14)] $\geq 0$. Here $\left(\dot{\tau}_{\mathbf{k}}^{\dot{c}}\right) \dot{\lambda}=1_{\text {corresponds to }(\dot{\gamma})} \dot{\lambda}=1_{1}=\partial \mathrm{f}_{1}^{(\mathrm{n})} / \partial \tau$ (eqn. (9)), and $\dot{\tau}_{\mathbf{k}}=\hat{\sigma}: \mathbf{G}_{\mathbf{k}}$. Thus $0 \leq \lambda<\infty$ unless there is no global hardening, i.e. the denominator in (14) is negative or nil.

3.3. Limit predictions at large $n$ vs. classical and viscoplastic predictions

Let $\mathbf{D}$ be given and examine the limit of the predicted stress $\sigma^{(n)}$ and shear rate $\dot{\gamma}^{(n)}$ as $n \rightarrow \infty$. From the maximum work principle (10) and the uniform proximity of $f^{(n)}(\sigma)$ and $f(\sigma)$ with $f(\sigma) \leq f^{(n)}(\sigma)$, it first follows that the work function of the regular form, $\dot{W}^{(n)}(D)$, tends (uniformly in D) towards the classical one $\dot{W}^{(\infty)}(D)$, as $n \rightarrow \infty$. Since $\sigma^{(n)}$ obviously has a limit $\sigma^{(\infty)}(\mathrm{D})$ (due to the monotonic evoluion of the yield surface $\Sigma^{(n)}$ as $n$ increases), this limit must thus satisfy $\sigma^{(\infty)}(\mathbf{D}): \mathbf{D}=$ $\dot{W}^{(\infty)}(D)$, i.e. it is associated with $D$ in the sense of the classical yield surface $\sum$. Hence, in the general case where $D$ is (strictly) within the cone of normals to $\sum$ at a corner, $\sigma^{(\infty)}(D)$ is the stress at that corner. It is less obvious to guess which associated stress is obtained, when $D$ is normal to a ridge, face, ..., of $\Sigma$. Moreover, for any shear rate vector $j^{(\infty)}$ which is a possible classical solution, i.e. which obeys the classical minimum work principle (12), or $\dot{\mathrm{W}}^{(\infty)}(\mathrm{D})=\dot{\mathrm{W}}_{2}^{(\infty)}\left(\dot{\boldsymbol{\gamma}}^{(\infty)}\right)$, we have thus

$$
\dot{\mathrm{W}}^{(\mathrm{n})}(\mathbf{D})=\dot{\mathrm{W}}_{2}^{(\mathrm{n})}\left(\dot{\gamma}^{(\mathrm{n})}\right) \rightarrow \dot{\mathrm{W}}_{2}^{(\infty)}\left(\dot{\gamma}^{(\infty)}\right)=\dot{\mathrm{W}}^{(\infty)}(\mathbf{D}), \mathrm{n} \rightarrow \infty
$$

Using the above argument with $\dot{\boldsymbol{\gamma}}, \tau, f_{1}^{(n)}$ and $f_{1}$ in the place of $D$, $\sigma, f^{(n)}$ and $f$, and substituting eqn. (13) to eqn. (10), it is found that the 
$\dot{\mathrm{W}}_{2}{ }^{(\mathrm{n})}(\dot{\boldsymbol{\gamma}})$ function tends, uniformly in $\dot{\boldsymbol{\gamma}}$, towards the function $\dot{\mathrm{W}}_{2}(\dot{\gamma})=\dot{\mathrm{W}}_{2}^{(\infty)}(\dot{\gamma})$ (eqn. 6).

Now suppose that the classical form leaves no ambiguity in $\dot{\gamma}^{(\infty)}(D)$ (this holds for any $D$ if the set of critical shear stresses is generic, i.e. if no corner of $\sum$ belongs to more than 5 critical hyperplanes ${ }^{4}$ ). It follows then from (15) that $\dot{\gamma}^{(n)}$ tends towards $\dot{\gamma}^{(\infty)}$ as $n \rightarrow \infty$ [otherwise one could assume, by extraction, that $\dot{\gamma}^{(n)} \rightarrow \dot{\gamma}^{\prime} \neq \dot{\gamma}^{(\infty)}$, whence $\dot{\mathrm{W}}_{2}^{(\mathrm{n})}\left(\dot{\gamma}^{(\mathrm{n})}\right) \rightarrow \dot{\mathrm{W}}_{2}^{(\infty)}\left(\dot{\gamma}^{\prime}\right)$ from the uniform proximity of $\dot{\mathrm{W}}_{2}^{(\mathrm{n})}$ and $\dot{\mathrm{W}}_{2}^{(\infty)}$. But $\dot{W}_{2}^{(\infty)}(\dot{\gamma})>\dot{W}_{2}^{(\infty)}\left(\dot{\gamma}^{(\infty)}\right)$ from the uniqueness of $\dot{\gamma}^{(\infty)}$ : contradiction with (15)].

This essential property of the proposed regular form is hence not true for the visco-plastic (VP) regularization used in 5-7 which also depends on an exponent $n$. Indeed, the comparison between the formulae in 5-7 and (9) gives :

$$
\left(\dot{\gamma}_{k}^{(n)}\right)_{V P}=\alpha(D, n) \tau_{k}\left(\dot{\gamma}_{k}^{(n)}\right)_{\text {proposed }}
$$

where the scaling factor $\alpha$ does not depend on the slip system $k$. As $\mathrm{n} \rightarrow \infty, \tau_{\mathbf{k}}$ tends towards $\tau_{\mathbf{k}}^{\mathrm{c}}$ for that systems which the classical form predicts to be active $\left(\dot{\gamma}_{k}^{(\infty)} \neq 0\right)$, for the viscoplastic regular form 7 as well as for the present one, since $\sigma^{(n)} \rightarrow \sigma^{(\infty)}$. Thus the limits of $\left(\dot{\gamma}^{(n)}\right)_{\text {VP }}$ and $\left(\dot{\gamma}^{(\mathrm{n})}\right)_{\text {proposed }}$ are not proportional, which means that $\left(\dot{\gamma}^{(\mathrm{n})}\right)_{\mathrm{VP}}$ does not tend towards $\dot{\gamma}^{(\infty)}$ [unless if the $\tau_{\mathrm{k}}^{\mathrm{c}}$ 's are all equal : but then $\dot{\gamma}^{(\infty)}$ is generally ambiguous]. This is not unnatural, since the regular yield criterion (8) differs from the VP stress potential 5,7 .

\section{CONCLUSIONS}

1) The proposed regular Schmid law consists in "rounding off the corners" from the very expression of the law. It solves ambiguity problems in crystal plasticity whenever they arise, without assuming a rate-sensitivity or a particular hardening matrix. However it is easy to adapt the proposed form so as to take into account the rate-sensitivity 10 in a different way from ${ }^{5-8}$. This leaves the shear rate ratios unchanged.

2) Straightforward procedures have been proposed for implementing this regularization in the various polycrystal models. 
3) The well-foundedness of the assumed normality in $\tau$-space (eqn. (9)) is confirmed by the result that all the obtained predictions tend towards the classical ones whenever these latter are unambiguous -which is the generic case, even though the simple "Taylor hardening" is then excluded for the classical form. In contrast, the visco-plastic (power-law) predictions for the shear rates do not tend towards the classical ones for unequal critical shear stresses. However, the proposed and visco-plastic regularizations tend towards the same limit for Taylor hardening. This justifies the use of the visco-plastic (power-law) regularization for solving ambiguities in that case.

\section{References}

1. B. Jaoul : Etude de la plasticité et applications aux métaux (Dunod, Paris 1965).

2. M. Renouard and M. Wintenberger, C.R. Acad. Paris, B283, 237 (1976) and B290, 403 (1980).

3. U.F. Kocks and H. Chandra, Acta Metall., 30, 695 (1982).

4. P. van Houtte, Textures of Materials (C.M. Brakman et al., edrs. ; Netherl. Soc. Mater. Sc., Zwijndrecht 1984), p. 7.

5. J.W. Hutchinson, Proc. Roy. Soc., A348, 101 (1976).

6. R.J. Asaro, A. Needleman, Acta Metall., 33, N $^{\circ}$ 6, 923 (1983).

7. L.S. Toth, P. Gilormini, J.J. Jonas, Acta Metall., 36, No 12, 3077 (1988).

8. G. Canova, C. Fressengeas, A. Molinari, U.F. Kocks, Acta Metall., 36; $\mathrm{N}^{\circ} 8,1961$ (1988).

9. T. Leffers, Textures of Materials (J.S. Kallend and G. Gottstein, edrs. ; Metall. Soc. AIME, Warrendale 1988), p. 273.

10. M. Arminjon, Lois de comportement homogénéisées pour la plasticité des polycristaux. Mémoire d'habilitation, Univ. Paris-Nord, Villetaneuse (1988).

11. J.F.W. Bishop, R. Hill, Phil. Mag., 42, 414 (1951).

12. H. Honneff, H. Mecking, Textures of Materials (G. Gottstein and K. Lücke, edrs. ; Springer, Berlin-Heidelberg-New York 1978), p. 265. 13. M. Arminjon, Acta Metall., 35, N$^{\circ} 3,615$ (1987).

14. M. Arminjon, C. Donadille, Mém. Et. Sc. Revue Métall., 87, $\mathrm{N}^{\circ} 6$, 359 (1990).

15. R. Hill, J. Mech. Phys. Solids, 14, 95 (1966).

16. A. Hihi, M. Berveiller, A. Zaoui, J. Méc. Th. Appl., 4, N 2, 201 (1985). 